\title{
Nuevos registros de helechos (Pteridophyta) para Honduras, Parque Nacional La Tigra y Reserva Biológica Misoco.
}

\author{
Ruth K. Hernández-Cibrián', Alexander Rojas-Alvarado² y Rony Moreno³ \\ 1Universidad Nacional Autónoma de Honduras (UNAH), Tegucigalpa. E-mail: rkhcibrian@gmail.com \\ 2Universidad Nacional de Costa Rica, Heredia (UNA), Costa Rica. e-mail: alfrojasa@yahoo.com. \\ ${ }^{3}$ Instituto Nacional de Conservación y Desarrollo Forestal, Áreas Protegidas y Vida Silvestre (ICF), Tegucigalpa, Honduras.
}

Correspondencia

A. Rojas-Alvarado

e-mail: alfrojasa@yahoo.com

Recibido: 9 junio 2017

Aceptado: 29 agosto 2017

Publicado on-line: diciembre 2017

\section{Resumen}

El Parque Nacional La Tigra (PNLT) y la Reserva Biológica Misoco, al poseer el ecosistema característico de los bosques nublados, son un reservorio importante de biodiversidad y específicamente de helechos, ambas áreas protegidas se encuentran fuertemente presionadas por las actividades antropogénicas.

Durante el proceso de investigación se realizó la colecta de especies en el Parque Nacional La Tigra el 05 de julio y en la Reserva Biológica Misoco del 09 al 11 de octubre de 2012 y la revisión de especímenes en los herbarios Universidad Nacional Autónoma de Honduras, Herbario Cyril Nelson (TEFH), Herbario Paul C. Standley, Universidad Zamorano (EAP), Herbario Nacional de México (MEXU), Missouri Botanical Garden (MO) y la base de datos Trópicos del Missouri Botanical Garden (MO), para obtener registros previos. Los especímenes fueron determinados y distribuidos a los herbarios CR, EAP y TEFH como testigos. Finalmente se obtuvieron un total de cinco nuevos registros para Honduras, 34 nuevos registros para el Parque Nacional La Tigra y 46 para la Reserva Biológica Misoco, aumentando así el conocimiento sobre los Pteridofitas en Honduras.

Palabras clave: Helechos, Honduras, La Tigra, Misoco, novedades corológicas.

\begin{abstract}
New records of ferns (Pteridophyta) from Honduras, La Tigra National Park, and Misoco Biological Reserve.

The National Park La Tigra (PNLT) and the Misoco Biological Reserve, possessing the ecosystem characteristic of cloud forests, are an important reservoir of biodiversity and specifically of ferns, both protected areas are strongly pressured by anthropogenic activities.

During the research process, the collection of species was carried out in $\mathrm{La}$ Tigra National Park on July 5 and in the Misoco Biological Reserve from 9 to 11 October 2012 and the review of specimens in the herbaria National Autonomous University of Honduras, Cyril Nelson Herbarium (TEFH), Herbarium Paul C. Standley, Zamorano University (EAP), National Herbarium of Mexico (MEXU), Missouri Botanical Garden (MO), and the Missouri Botanical Garden (MO) Tropics database for prior records. Specimens were determined and distributed to the herbaria CR, EAP and TEFH as controls. Finally, a total of five new records were obtained for Honduras, 34 new records for La Tigra National Park and 46 for Reserva Biológica Misoco, thus increasing knowledge about Pteridophytes in Honduras.
\end{abstract}

Key words: Ferns, Honduras, La Tigra, Misoco, chorological novelties 


\section{Introducción}

Honduras posee una gran biodiversidad de plantas que aún no ha sido estudiada o inventariada en su totalidad. Existen a nivel nacional 91 áreas protegidas dentro de las cuales se destacan el Parque Nacional La Tigra y La Reserva Biológica de Misoco, las cuales son objeto del presente estudio y que fueron declaradas mediante Decreto No. 87-87 "Cuya finalidad es la protección de los bosques Nublados como reservorio y fuente de biodiversidad y agua (Congreso Nacional de Honduras, 1987).

El bosque nublado del neotrópico se caracteriza por la presencia constante de nubes o niebla en movimiento, que aumenta la cantidad de humedad en la atmósfera y el volumen de agua depositada sobre la vegetación y el suelo. La vegetación de los bosques nublados es compleja, se encuentra alta riqueza de especies, donde pocas son abundantes y muchas especies son raras o poco frecuentes (Jiménez, 2009).

El Parque Nacional La Tigra (PNLT), se encuentra ubicado aproximadamente a $12 \mathrm{Km}$ de la ciudad de Tegucigalpa, en el Departamento de
Francisco Morazán, tiene un área total de 24,040 hectáreas, con un núcleo de 7.571 ha y una zona de amortiguamiento de 16,469 ha (Tribunal Superior de Cuentas, 2012).

La Tigra tiene cuatro zonas de vida (basada en la clasificación de Holdridge 1962) que son: a) El bosque húmedo subtropical (bh-S), donde se encuentra la asociación denominada Ocotal, ubicada por debajo de los $1.300 \mathrm{~m}, \mathrm{~b}$ ) El bosque húmedo montano bajo subtropical (bhMBS), donde se encuentra la asociación pino y liquidámbar ubicada entre $1,300 \mathrm{~m}$ y $1.800 \mathrm{~m}$ y el bosque nublado de maderas duras ubicado arriba de los $1.800 \mathrm{~m}, \mathrm{c}$ ) El bosque muy húmedo montano bajo subtropical (bmh-MBS) y d) El bosque seco subtropical, bs-S (Villeda, 2013). Fig. 1

La Reserva Biológica de Misoco (RBM), se sitúa aproximadamente a $100 \mathrm{Km}$, al suroeste de Tegucigalpa; se encuentra entre los municipios de Guaimaca y Orica en el Departamento de Francisco Morazán y los municipios de Concordia y Guayape en el Departamento de Olancho, tiene un área total de 4,572.36 ha (ICF, 2016).

En la RBM se identifican los siguientes ecosistemas (según el sistema de clasificación

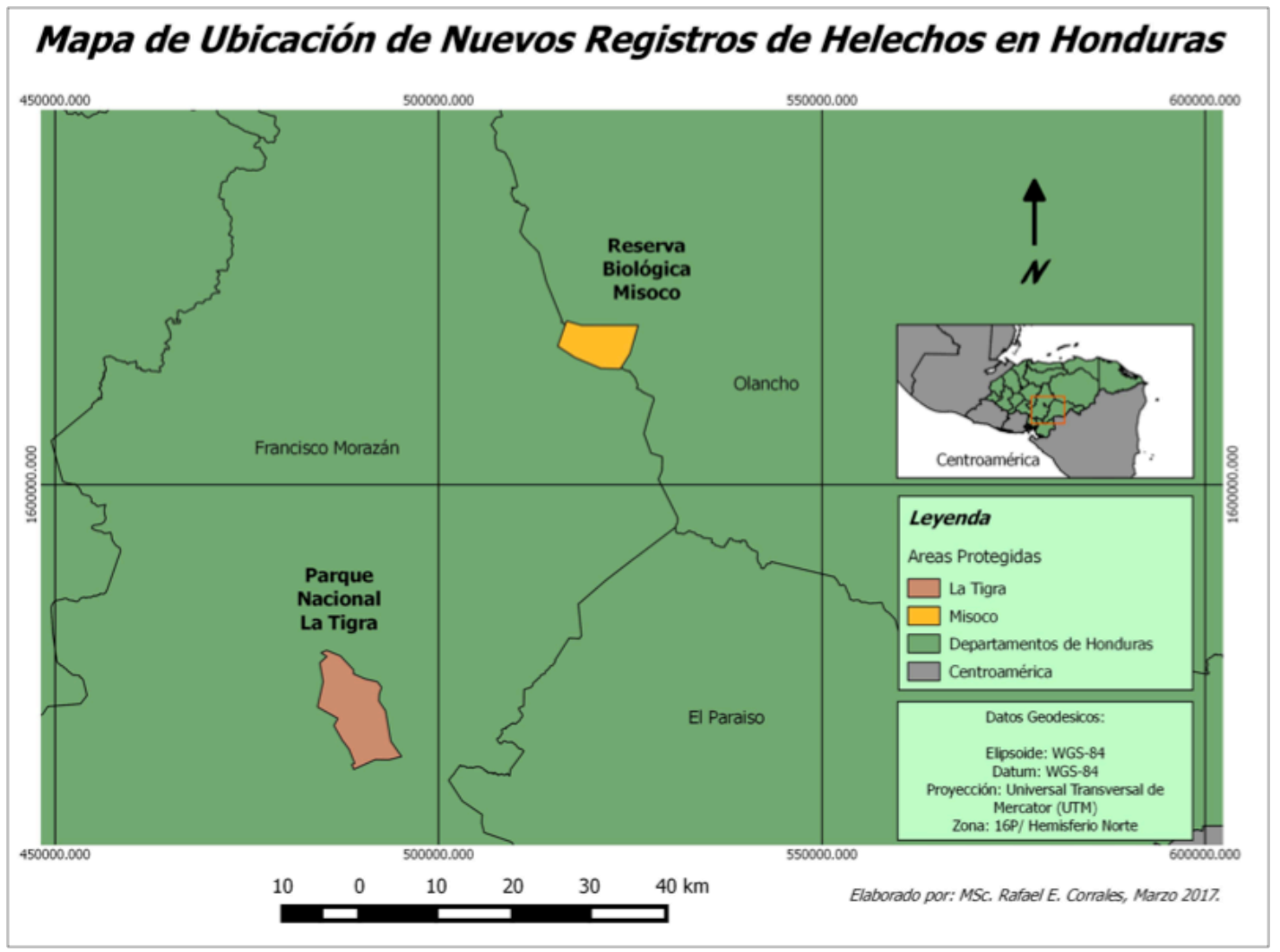

Figura 1. Ubicación de áreas protegidas de los nuevos registros de helechos para Honduras. Figure 1. Location of protected areas of new ferns records from Honduras. 
UNESCO, 1973): a) Bosque latifoliado Montano Superior, tiene un área de 2.097.6948 Ha, donde se encuentran especies como Pino (Pinus pseudostrobus Lindl.), Roble (Quercus oleoides Schltdl.\&Cham.),Araliaceae(Oreopanaxxalapensis (Kunth) Decne. \& Planch.), Anacardiaceae (Mauria sessiliflora Standl.) y algunos helechos y palmas abundantes, b) Bosque latifoliado altimontano, ubicado en la zona núcleo de la reserva a una altura que va desde 1, 800 hasta 2,143 m en el Cerro el Volcán. Es común encontrar especies como Tapa tamal (Alchornea latifolia Sw.) y araliáceas como Dendropanax sp., c) Bosque acicuifoliado sub montano, ubicado aproximadamente $700-900 \mathrm{~m}$ y la especie predominante es el Pino (Pinus oocarpa Schiede ex Schltdl.), d) Bosque acicuifoliado montano inferior, Ubicado entre los 900 a 1,200 m, la especie más común es el Pino (Pinus oocarpa) y e) Sistema agropecuario, Comprende una área de 1.361.58 has, forma parte de casi toda la zona de amortiguamiento que va desde los 800 a 1,100 m, fuertemente influenciada (en todos los municipios) por los cultivos de subsistencia como café, maíz, frijoles y bananos (ICF, 2015).

Las áreas protegidas en Honduras están siendo fuertemente presionadas por el avance de la agricultura y la ganadería por lo que es de suma importancia realizar estudios con la finalidad de conocer las especies que existen en ellas y contribuir así a generar información que ayude a realizar una mejor gestión de estas áreas protegidas, especialmente las del presente estudio.

Es importante resaltar que existen varias publicaciones y estudios sobre los helechos y licopodios de Honduras. El estudio más reciente sobre helechos fue realizado en el Parque Nacional Montaña de Celaque (PNMC), en el cual se reporta el descubrimiento de nueve registros de helechos para Honduras de un total de 210 (Rojas, 2012).

En el PNLT el primer estudio de vegetación fue realizado por Cruz \& Erazo (1977), en el cual reporta un total de 33 especies de helechos, posteriormente Hernández et al., (2005 y 2006) realizaron dos estudios sobre la diversidad de helechos en los senderos La Esperanza (54 especies) y La Cascada (55 especies).

Hernández (2012) realiza el primer inventario de helechos para la RBM en el cual reporta un total de 46 especies.

\section{Metodología}

La investigación se realizó en el Parque Nacional La Tigra (PNLT) y la Reserva Biológica Misoco (RBM), e involucró colecta de especímenes y revisión de material en los herbarios Universidad Nacional Autónoma de Honduras, Herbario Cyril Nelson (TEFH), Herbario Paul C. Standley, Universidad Zamorano (EAP), Herbario Nacional de México (MEXU) y Missouri Botanical Garden (MO).

El trabajo de campo en el PNLT, se realizó entre el 05 de julio de 2012 y consistió en el recorrido de la calle principal $1 \mathrm{Km}$ (cerca del centro de visitantes) y el sendero la Cascada (8 Km y reversa) del Parque Nacional La Tigra (1.700 m hasta 2.020 $\mathrm{m})$, obteniendo un total de 67 especímenes y 55 especies.

El trabajo de campo en la RBM se realizó del 09 al 11 de octubre de 2012, en tres diferentes lugares a) el sendero Los Olingos ( $2.5 \mathrm{Km})$ aprox. a $1729 \mathrm{~m}$, próximo a la Comunidad de Monte Galán, Municipio de Guaimaca, Departamento de Francisco Morazán, b) Comunidad de Batideros Orica $(1 \mathrm{Km})$ aprox. a $1674 \mathrm{~m}$, Departamento de Francisco Morazán y c) Comunidad Ojo de Agua Municipio de Concordia (1 Km) aprox. a $1517 \mathrm{~m}$, en el Departamento de Olancho, en donde se colectó un total de 46 especímenes.

Es importante resaltar que los especímenes fueron recolectados y preservados de acuerdo con el método establecido por Lorea y Riba (1990), y distribuidos en los siguientes herbarios: a) Universidad Nacional Autónoma de Honduras, Herbario Cyril Nelson (TEFH) b) Herbario Paul C. Standley, Universidad Zamorano (EAP) y c) Museo Nacional de Costa Rica (CR).

La determinación de los especímenes se hizo principalmente con base en los tratamientos de Flora Mesoamericana (Moran, Riba, 1995) y The Pteridophytes of Mexico (Mickel \& Smith, 2004).

\section{Resultados}

En la realización de ambas investigaciones se obtuvo un total de cuatro nuevos registros para Honduras: cinco para el Parque Nacional La Tigra y cuatro para la Reserva Biológica Misoco.

En el PNLT se obtuvieron un total 12 familias, la más común fue Polypodiaceae con 10 géneros (Alansmia M. Kessler, Moguel, Sundue \& Labiak, Ascogrammitis Sundue, Campyloneurum C. Presl, Cochlidium Kaulf., Lellingeria A.R. Sm. \& R.C. Moran, Melpomene A.R. Sm. \& R.C. Moran, Moranopteris R.Y. Hirai \& J. Prado, Mycopteris Sundue, Polypodium L. y Stenogrammitis Labiak), seguida por la familia Hymenophyllaceae y Dryopteridaceae cada uno con tres géneros. Finalmente se obtuvo un total de 34 nuevos registros para el Parque Nacional La Tigra y 55 especies colectadas. 
Para la RBM se obtuvieron un total 28 géneros, 15 familias, la más común fue Polypodiaceae con 7 géneros (Campyloneurum, Melpomene, Niphidium J. Sm., Pecluma M.G. Price, Phlebodium (R. Br.) J. Sm., Pleopeltis Humb. \& Bonpl. ex Willd. y Polypodium), seguida por las familias Cyatheaceae, Dryopteridaceae, Hymenophyllaceae, Pteridaceae y Thelypteridace con dos géneros respectivamente. Es importante resaltar que este es el primer inventario de helechos realizado en la reserva. Finalmente se obtuvo un total de 45 especies.

\section{Nuevos Registros para Honduras}

Asplenium rutaceum (Willd.) Mett., Abh. Senckenberg. Naturf. Ges. 3: 173 (1859). Aspidium rutaceum Willd., Sp. PI. 5: 266 (1810). Lectotipo. (Designado por Morton \& Lellinger, 1966): Plumier, Traité Foug. Amér, t. 57 (1705).

Distribución. S. México, Guatemala, Honduras, Nicaragua, Costa Rica, Panamá, Colombia, Venezuela, Guyana, Surinam, Ecuador, Galápagos, Perú, Bolivia, Jamaica y La Española.

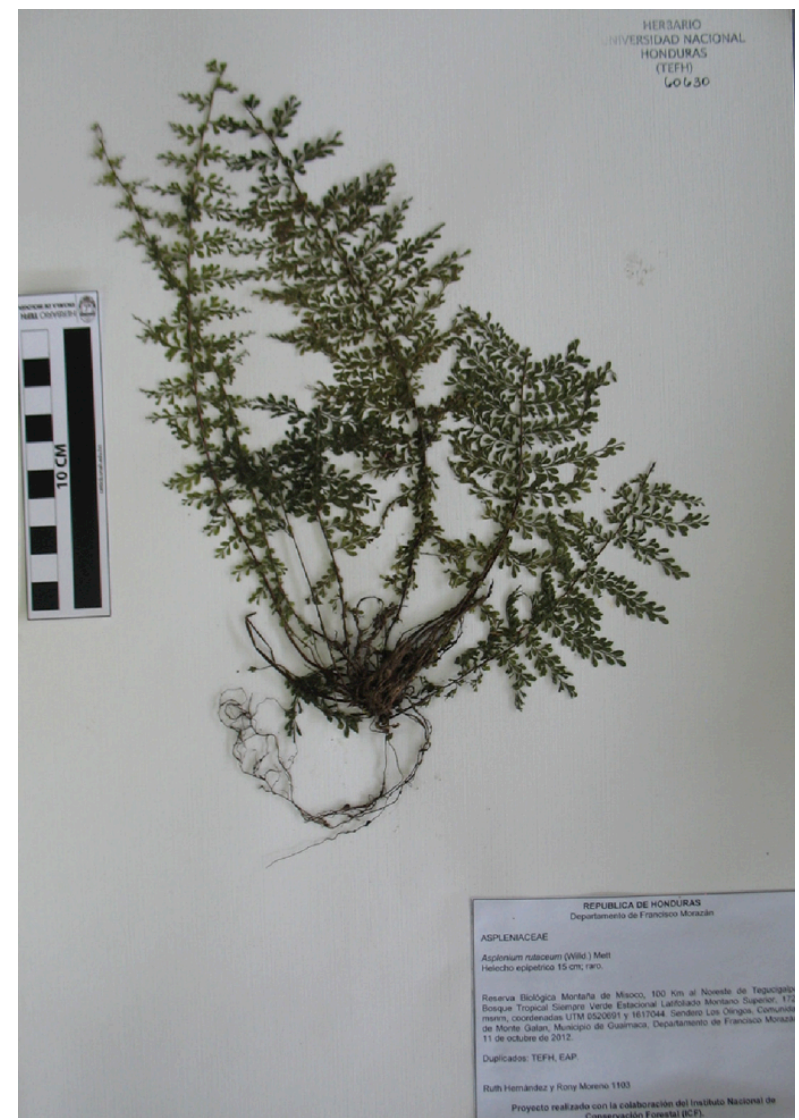

Figura 2. Asplenium rutaceum (R. Hernández \& R. Moreno 1103, TEFH). Figure 2. Asplenium rutaceum ( $R$. Hernández \& R. Moreno 1103, TEFH).
Material de nueva distribución. HONDURAS. Francisco Morazán: Reserva Biológica Montaña de Misoco, $100 \mathrm{Km}$ al Noreste de Tegucigalpa. sendero Los Olingos, comunidad de Monte Galán, $14^{\circ} 37^{\prime} 36^{\prime \prime} \mathrm{N}, 8^{\circ} 48^{\prime} 28^{\prime \prime} \mathrm{W}, 1729 \mathrm{~m}, 11$ oct 2012, $R$. Hernández \& R. Moreno 1103 (EAP, TEFH) (fig. 2).

Comentarios. Esta especie se asemeja a Asplenium maxonii Lellinger en los ejes atropurpúreos, grado de división de la lámina y ápice cirrado terminando en una yema. Se diferencia porque $A$. rutaceum tiene los últimos segmentos libres elípticos con el ápice agudo o apiculado (vs. obovados con el ápice obtuso o redondeado en $A$. maxonii), además por lo general $A$. maxonii suele tener las pínnulas acroscópicas basales con los segmentos parcialmente fusionados (vs. libres en A. rutaceum). Su aparición en Honduras era de esperarse debido a la amplia distribución que esta especie tiene en el neotrópico.

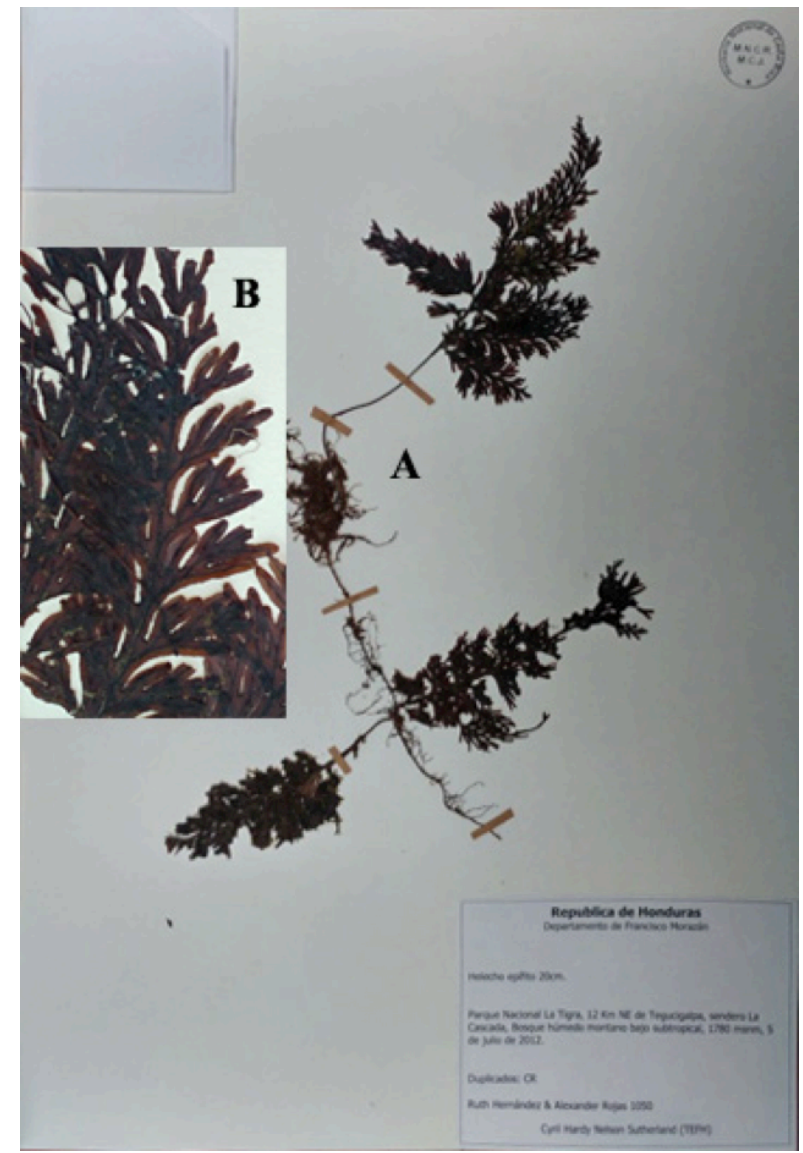

Figura 3. Hymenophyllum consanguineum (R. Hernández \& A. Rojas 1050, CR). A. Hábito. B. Detalle de la lámina. Figure 3. Hymenophyllum consanguineum (R. Hernández \& A. Rojas 1050, CR). A. Habit. B. Blade detail. 
Hymenophyllum consanguineum C.V. Morton, Contr. U.S. Natl. Herb. 29: 163. (1947). Tipo: Panamá, W. Maxon 5624 [Holotipo US; Isotipos: $\mathrm{BM}, \mathrm{GH}, \mathrm{LE}, \mathrm{NY}, \mathrm{P}, \mathrm{S}]$.

Distribución. Honduras, Costa Rica, Panamá y Venezuela.

Material de nueva distribución. HONDURAS. Francisco Morazán: Parque Nacional La Tigra, $12 \mathrm{Km}$ NE de Tegucigalpa, sendero La Cascada, $14^{\circ} 12^{\prime} 20^{\prime \prime N}, 87^{\circ} 07^{\prime} 35^{\prime \prime} \mathrm{W}, 1780 \mathrm{~m}, 5$ jul 2012, $R$. Hernández \& A. Rojas 1050 (CR, EAP, TEFH) (fig. 3).

Comentarios: Esta especie se puede caracterizar por tejido laminar peloso en las venas y margen (no en el tejido laminar), estípite 0.3-1 $\mathrm{mm}$ de diámetro y no alado, y raquis alado hasta la base. Esta ampliación de distribución hace que Honduras sea su límite Norte.

Hymenophyllum maxonii Christ ex C.V. Morton, Contr. U.S. Natl. Herb. 29: 165. (1947). Tipo:

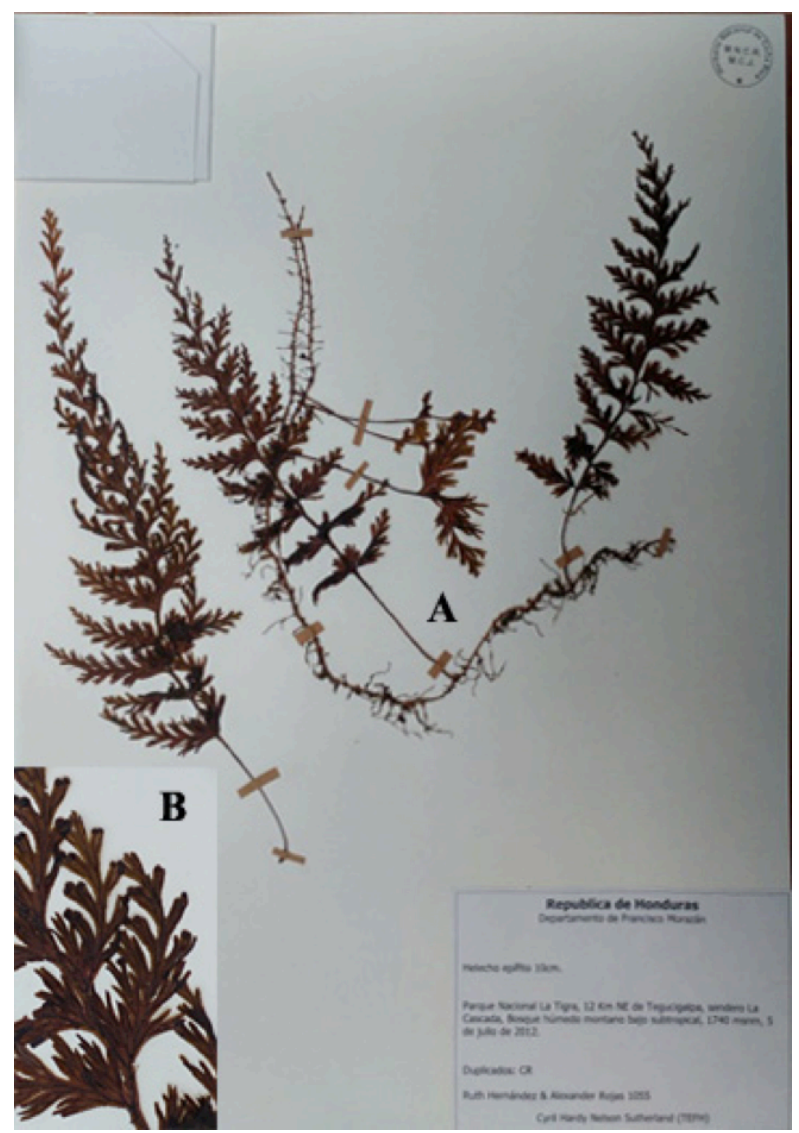

Figura 4. Hymenophyllum maxonii (R. Hernández \& A. Rojas 1055, CR). A. Hábito. B. Detalle de la lámina. Figura 4. Hymenophyllum maxonii (R. Hernández \& A. Rojas 1055, CR). A. Habit. B. Blade detail.
Guatemala, W. Maxon \& R. Hay 3326 (holotipo: US; Isotipo: GH).

Distribución: S. México, Guatemala y Honduras.

Material de nueva distribución. HONDURAS. Francisco Morazán: Parque Nacional La Tigra, $12 \mathrm{Km}$ NE de Tegucigalpa, sendero La Cascada, bosque húmedo montano bajo subtropical, $14^{\circ} 12^{\prime} 20^{\prime \prime} \mathrm{N}, 87^{\circ} 07^{\prime} 35^{\prime \prime W}, 1740 \mathrm{~m}, 5$ jul 2012, R. Hernández \& A. Rojas 1055 (CR, EAP, TEFH) (fig. 4).

Comentarios: Esta especie se diferencia de Hymenophyllum consanguineum por tricomas del margen bifurcados o estrellados, de $\mathrm{H}$. hirsutum (L.) Sw. por pecíolo exhalado y de $H$. trapezoidale Liebm. por raquis alado hasta la base.

Polypodium puberulum Schltdl. \& Cham., Linnaea 5: 607 (1830). Isotipo: México, Schiede \& F. Deppe 747 (Isotipo B). Polypodium

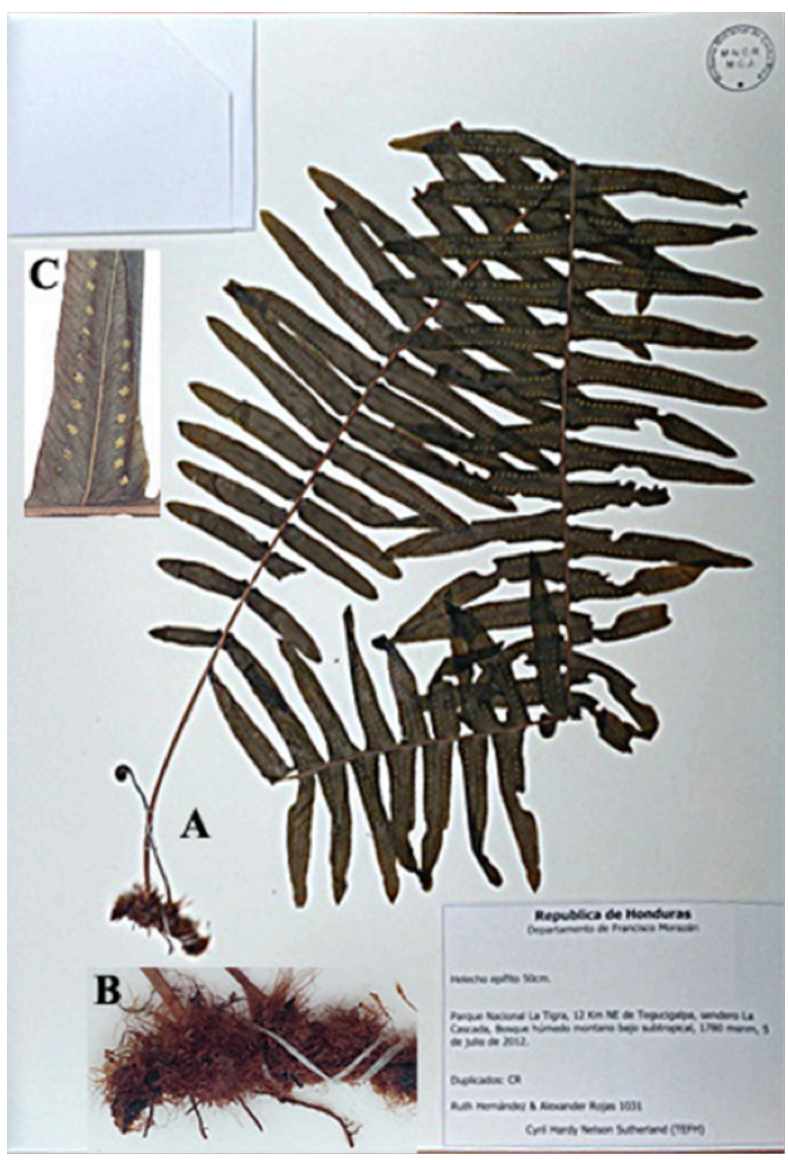

Figura 5. Polypodium puberulum (R. Hernández \& A. Rojas 1031, CR). A. Hábito. B. Rizoma. C. Detalle de pinna. Figure 5. Polypodium puberulum (R. Hernández \& A. Rojas 1031, CR). A. Habit. B. Rhizome. C. Pinna detail. 


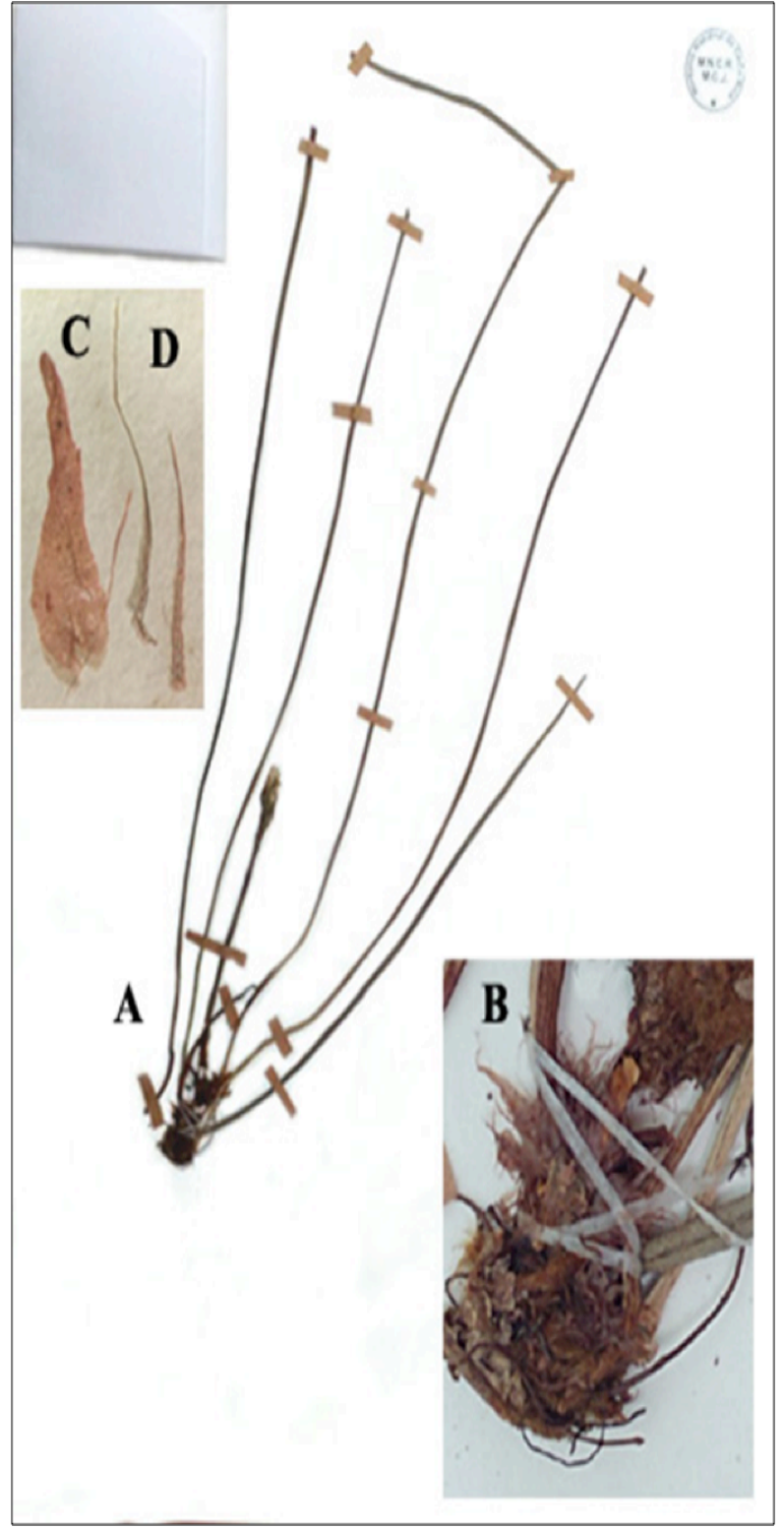

Figura 6. Vittaria costaricensis (A. Rojas et al. 10067, CR). A. Hábito. B. Rizoma. C. Escama del rizoma. Vittaria lineata. D. Escamas del rizoma. Figura 6. Vittaria costaricensis (A. Rojas et al. 10067, CR). A. Habit. B. Rhizome. C. Rhizome scale. Vittaria lineata. D. Rhizome scales.

ehrenbergianum Klotzsch, Linnaea 20: 381. (1847). Tipo: México, Ehrenberg s.n. (B; Isotipo $\mathrm{S}$, photo, US).

Distribución: México, Honduras, El Salvador.

Material de nueva distribución. HONDURAS. Francisco Morazán: Parque Nacional La Tigra, $12 \mathrm{Km}$ NE de Tegucigalpa, sendero La Cascada, bosque húmedo montano bajo subtropical, $14^{\circ} 12^{\prime 2} 20^{\prime \prime} \mathrm{N}$, 8707'35’W, 1780 m, 5 jul 2012, R. Hernández \& A. Rojas 1031, (CR, EAP,TEFH) (fig. 5).
Comentarios: Según Morán (1995) Polypodium puberulum difiere de $P$. hartwegianum Hook. por soros redondeados y peciolo 0.3-1 veces el largo de la lámina. Sin embargo, $P$. hartwegianum puede tener peciolo largo o corto al igual que $P$. puberulum, lo que si las distingue son las escamas del rizoma pardo rojizas en $P$. puberulum (vs. amarillo pálido, al menos terminalmente en $P$. harwegianum) y frondas más grandes con pinnas más anchas en $P$. puberulum.

Vittaria costaricensis Lellinger, Proc. Biol. Soc. Washington 98(2): 389, f. 18. 1985. Tipo: Costa Rica, Guanacaste, El Silencio, near Tilarán, ca. 750 m, P. Standley \& J. Valerio 44805 (Holotipo: US (foto, CR-131785); Isotipo: GH).

Distribución: Honduras, Costa Rica.

Material de nueva distribución. HONDURAS. Lempira: Parque Nacional Montañas de Celaque, sendero Principal, entre campamento El Naranjo y el cruce del sendero rumbo al cerro Las Minas, $14^{\circ} 32^{\prime} 36^{\prime \prime} \mathrm{N}, 88^{\circ} 40^{\prime} 57^{\prime \prime} \mathrm{W}, 2575-2700 \mathrm{~m}, 28$ jun 2012, A. Rojas et al. 10067 (CR, EAP, TEFH) (fig. $6)$.

Comentarios: Por mucho tiempo Vittaria costaricensis ha sido considerada como sinónimo de V. lineata (L.) Sm. por sus frondas largamente lineares, pero esta semejanza morfológica no refleja una semejanza evolutiva, porque sus escamas son escasamente clatradas, lanceoladas y anaranjadas a pardo-grisáceas, revelando probablemente similitud con especies como $\mathrm{V}$. graminifolia Kaulf.

Nuevos registros para el Parque Nacional La Tigra:

Alansmia senilis (Fée) Moguel \& M. Kessler (R. Hernández \& A. Rojas 1003, CR, EAP, TEFH). Ubicación. Sendero La Cascada.

Alsophila tryoniana (Gastony) D.S. Conant (R. Hernández \& A. Rojas 1063, EAP, CR, TEFH). Ubicación. Sendero principal.

Campyloneurum angustifolium (Sw.) Fée (R. Hernández \& A. Rojas 1033, EAP, CR, TEFH). Ubicación. Sendero principal.

Cochlidium serrulatum (Sw.) L.E. Bishop (R. Hernández \& A. Rojas 1044, EAP, CR, TEFH). Ubicación. Sendero La Cascada.

Ctenitis grisebachii (Baker) Ching (R. Hernández \& A. Rojas 1013, EAP, CR, TEFH). Ubicación. Sendero La Cascada.

Cyathea bicrenata Liebm. (R. Hernández \& A. Rojas 1059, EAP, CR, TEFH).

Ubicación. Sendero principal

Dennstaedtia dissecta (Sw.) T. Moore (R. Hernández \& A. Rojas 1040, EAP, CR, TEFH). 
Ubicación. Sendero La Cascada.

Diplazium urticifolium var. mesoamericanum A. Rojas (R. Hernández \& A. Rojas 1020, EAP, CR, TEFH). Ubicación. Sendero La Cascada.

Elaphoglossum glaucum T. Moore (R. Hernández \& A. Rojas 1037, EAP, CR, TEFH). Ubicación. Sendero La Cascada.

Elaphoglossum guatemalense (Klotzsch) T. Moore (R. Hernández \& A. Rojas 1036, .EAP, CR, TEFH). Ubicación. Sendero La Cascada.

Elaphoglossum mexicanum (E. Fourn.) A. Rojas (R. Hernández \& A. Rojas 1007, EAP, CR, TEFH). Ubicación. Sendero La Cascada.

Elaphoglossum paleaceum (Hook. \& Grev.) Sledge (R. Hernández \& A. Rojas 1061, EAP, CR, TEFH). Ubicación. Sendero principal.

Elaphoglossum petiolatum (Sw.) Urb. (R. Hernández \& A. Rojas 1039, EAP, CR, TEFH). Ubicación. Sendero La Cascada.

Elaphoglossum polypodium A. Rojas (R. Hernández \& A. Rojas 1016, EAP, CR, TEFH). Ubicación. Sendero La Cascada.

Hymenophyllum crispum Kunth (R. Hernández \& A. Rojas 1023, EAP, CR, TEFH). Ubicación. Sendero La Cascada.

Hymenophyllum hirsutum (L.) Sw. (R. Hernández \& A. Rojas 1002, EAP, CR, TEFH). Ubicación. Sendero La Cascada.

Hymenophyllum tegularis (Desv.) Proctor \& Lourteig (R. Hernández \& A. Rojas 1001, EAP, CR, TEFH). Ubicación. Sendero La Cascada.

Lycopodium clavatum L. (R. Hernández \& A. Rojas 1062, EAP, CR, TEFH). Ubicación. Sendero principal.

Macrothelypteris torresiana (Gaudich.) Ching (R. Hernández \& A. Rojas 1052, EAP, CR, TEFH). Ubicación. Sendero La Cascada.

Melpomene pilosissima (M. Martens \& Galeotti) A.R. Sm. \& R.C. Moran (R. Hernández \& A. Rojas 1045, EAP, CR, TEFH). Ubicación. Sendero La Cascada.

Mildella intramarginalis (Kaulf. ex Link) Trevis ( $R$. Hernández \& A. Rojas 1057, EAP, CR, TEFH). Ubicación. Sendero principal.

Moranopteris basiattenuata (Jenman) R.Y. Hirai \& J. Prado (R. Hernández \& A. Rojas 1047, EAP, CR, TEFH).

Ubicación. Sendero La Cascada.

Mycopteris semihirsuta (Klotzsch) Sundue (R. Hernández \& A. Rojas 1004, EAP, CR, TEFH). Ubicación. Sendero La Cascada.

Mycopteris subtilis (Kunze ex Klotzsch) Sundue ( $R$. Hernández \& A. Rojas 1005, EAP, CR, TEFH). Ubicación. Sendero La Cascada.

Polypodium pleurosorum Kunze ex Mett. (R. Hernández \& A. Rojas 1028, EAP, CR, TEFH). Ubicación. Sendero La Cascada.

Polystichum muricatum (L.) Fée (R. Hernández \& A. Rojas 1014, EAP, CR, TEFH). Ubicación. Sendero La Cascada.

Pteridium arachnoideum (Kaulf.) Maxon (R. Hernández \& A. Rojas 1043, EAP, CR, TEFH).
Ubicación. Sendero La Cascada.

Pteridium caudatum (L.) Maxon (R. Hernández \& A. Rojas 1027, EAP, CR, TEFH).

Ubicación. Sendero La Cascada.

Pteridium feei (W. Schaffn. ex Fée) Faull (R. Hernández \& A. Rojas 1029, EAP, CR, TEFH). Ubicación. Sendero La Cascada.

Selaginella silvestris Aspl. (R. Hernández \& A. Rojas 1017, EAP, CR, TEFH).

Ubicación. Sendero La Cascada.

Sticherus bifidus (Willd.) Ching (R. Hernández \& A. Rojas 1030, EAP, CR, TEFH).

Ubicación. Sendero La Cascada.

Christella dentata (Forssk.) Brownsey \& Jermy (R. Hernández \& A. Rojas 1049, EAP, CR, TEFH). Ubicación. Sendero La Cascada.

Thelypteris pilosula (Klotzsch \& H. Karst. ex Mett.) R.M. Tryon (R. Hernández \& A. Rojas 1038, EAP, CR, TEFH).

Ubicación. Sendero La Cascada.

Trichomanes krausii Hook. et Grev. (R. Hernández \& A. Rojas 1034, EAP, CR, TEFH).

Ubicación. Sendero La Cascada.

Nuevos registros de helechos para la Reserva Biológica Misoco

Adiantum andicola Liebm. (R. Hernández y R. Moreno 1079, EAP, TEFH).

Ubicación. Comunidad de Ojo de Agua, Concordia, Departamento de Olancho.

Adiantum concinnum Humb. \& Bonpl. ex Willd. (R. Hernández y R. Moreno 1068, EAP, TEFH). Ubicación. Comunidad de Ojo de Agua, Concordia, Departamento de Olancho.

Adiantum feei T. Moore ex Fée (R. Hernández y R. Moreno 1084, EAP, TEFH).

Ubicación. Comunidad de Batideros, Municipio de Orica, Departamento de Francisco Morazán.

Alsophila salvinii Hook. in Hook. et Baker (R. Hernández y R. Moreno 1095, EAP, TEFH).

Ubicación. Sendero Los Olingos, Comunidad de Monte Galán, Municipio de Guaimaca, Departamento de Francisco Morazán.

Arachniodes denticulata (Sw.) Ching (R. Hernández y R. Moreno 1099, EAP, TEFH).

Ubicación. Sendero Los Olingos, Comunidad de Monte Galán, Municipio de Guaimaca, Departamento de Francisco Morazán.

Asplenium abscissum Willd. (R. Hernández y R. Moreno 1089, EAP, TEFH).

Ubicación. Sendero Los Olingos, Comunidad de Monte Galán, Municipio de Guaimaca, Departamento de Francisco Morazán.

Asplenium auriculatum Sw. (R. Hernández y R. Moreno 1088, EAP, TEFH).

Ubicación. Sendero Los Olingos, Comunidad de Monte Galán, Municipio de Guaimaca, Departamento de Francisco Morazán.

Asplenium cuspidatum Lam. (R. Hernández y R. Moreno 1085, EAP, TEFH).

Ubicación. Comunidad de Batideros, Municipio de Orica, Departamento de Francisco Morazán. 
Asplenium radicans L. (R. Hernández y R. Moreno 1098, EAP, TEFH).

Ubicación. Sendero Los Olingos, Comunidad de Monte Galán, Municipio de Guaimaca, Departamento de Francisco Morazán.

Asplenium serra Langsd. et Fisch. (R. Hernández y R. Moreno 1073, EAP, TEFH).

Ubicación. Comunidad de Ojo de Agua, Concordia, Departamento de Olancho.

Blechnum divergens (Kunze) Mett. (R. Hernández y R. Moreno 1078, EAP, TEFH).

Ubicación. Comunidad de Ojo de Agua, Concordia, Departamento de Olancho.

Blechnum Iherminieri (Bory) C. Chr. (R. Hernández y R. Moreno 1090, EAP, TEFH).

Ubicación. Sendero Los Olingos, Comunidad de Monte Galán, Municipio de Guaimaca, Departamento de Francisco Morazán.

Blechnum schiedeanum (Schltdl. ex C. Presl) Hieron. (R. Hernández y R. Moreno 1091, EAP, TEFH).

Ubicación. Sendero Los Olingos, Comunidad de Monte Galán, Municipio de Guaimaca, Departamento de Francisco Morazán.

Botrychium underwoodianum Maxon (R. Hernández y R. Moreno 1077, EAP, TEFH).

Ubicación. Comunidad de Ojo de Agua, Concordia, Departamento de Olancho.

Campyloneurum angustifolium (Sw.) Fée (R. Hernández y R. Moreno 1081, EAP, TEFH).

Ubicación. Comunidad de Ojo de Agua, Concordia, Departamento de Olancho.

Cibotium regale Verschaff. et Lem. (R. Hernández y $R$. Moreno 1083, EAP, TEFH).

Ubicación. Comunidad de Batideros, Municipio de Orica, Departamento de Francisco Morazán.

Cyathea divergens var. tuerckheimii (Maxon) R.M. Tryon (R. Hernández y R. Moreno 1096, EAP, TEFH).

Ubicación. Sendero Los Olingos, Comunidad de Monte Galán, Municipio de Guaimaca, Departamento de Francisco Morazán.

Cyathea valdecrenata Domin (R. Hernández y R. Moreno 1101, EAP, TEFH).

Ubicación. Sendero Los Olingos, Comunidad de Monte Galán, Municipio de Guaimaca, Departamento de Francisco Morazán.

Dennstaedtia bipinnata (Cav.) Maxon (R. Hernández y R. Moreno 1092, EAP, TEFH).

Ubicación. Sendero Los Olingos, Comunidad de Monte Galán, Municipio de Guaimaca, Departamento de Francisco Morazán.

Diplazium donnell-smithii Christ (R. Hernández y R. Moreno 1110, EAP, TEFH).

Ubicación. Sendero Los Olingos, Comunidad de Monte Galán, Municipio de Guaimaca, Departamento de Francisco Morazán.

Diplazium ternatum Liebm. (R. Hernández y R. Moreno 1100, EAP, TEFH).

Ubicación. Sendero Los Olingos, Comunidad de Monte Galán, Municipio de Guaimaca, Departamento de Francisco Morazán.

Elaphoglossum latifolium (Sw.) J. Sm. (R. Hernández y R. Moreno 1086, EAP, TEFH).
Ubicación. Comunidad de Batideros, Municipio de Orica, Departamento de Francisco Morazán.

Elaphoglossum tectum (Humb. et Bonpl. ex Willd.) T. Moore (R. Hernández y R. Moreno 1111, EAP, TEFH). Ubicación. Sendero Los Olingos, Comunidad de Monte Galán, Municipio de Guaimaca, Departamento de Francisco Morazán.

Hymenophyllum polyanthos (Sw.) Sw. (R. Hernández y R. Moreno 1106, EAP, TEFH).

Ubicación. Sendero Los Olingos, Comunidad de Monte Galán, Municipio de Guaimaca, Departamento de Francisco Morazán.

Hymenophyllum trapezoidale Liebm. (R. Hernández y R. Moreno 1105, EAP, TEFH).

Ubicación. Sendero Los Olingos, Comunidad de Monte Galán, Municipio de Guaimaca, Departamento de Francisco Morazán.

Lophosoria quadripinnata (J.F. Gmel.) C. Chr. in Skottsb. (R. Hernández y R. Moreno 1108, EAP, TEFH).

Ubicación. Sendero Los Olingos, Comunidad de Monte Galán, Municipio de Guaimaca, Departamento de Francisco Morazán.

Macrothelypteris torresiana (Gaudich.) Ching (R. Hernández y R. Moreno 1109, EAP, TEFH). Ubicación. Sendero Los Olingos, Comunidad de Monte Galán, Municipio de Guaimaca, Departamento de Francisco Morazán.

Marattia excavata Underw. (R. Hernández y R. Moreno 1097, EAP, TEFH).

Ubicación. Sendero Los Olingos, Comunidad de Monte Galán, Municipio de Guaimaca, Departamento de Francisco Morazán.

Megalastrum pulverulentum (Poir.) A.R. Sm. \& R.C. Moran (R. Hernández y R. Moreno 1107, EAP, TEFH). Ubicación. Sendero Los Olingos, Comunidad de Monte Galán, Municipio de Guaimaca, Departamento de Francisco Morazán.

Melpomene anfractuosa (Kunze ex Klotzsch) A.R. Sm. et R. C. Moran (R. Hernández y R. Moreno 1075, EAP, TEFH).

Ubicación. Comunidad de Ojo de Agua, Concordia, Departamento de Olancho.

Niphidium crassifolium (L.) Lellinger (R. Hernández y R. Moreno 1071, EAP, TEFH).

Ubicación. Comunidad de Ojo de Agua, Concordia, Departamento de Olancho.

Pecluma alfredii (Rosenst.) M.G. Price (R. Hernández y R. Moreno 1072, EAP, TEFH).

Ubicación. Comunidad de Ojo de Agua, Concordia, Departamento de Olancho.

Phlebodium pseudoaureum (Cav.) Lellinger (R. Hernández y R. Moreno 1074, EAP, TEFH).

Ubicación. Comunidad de Ojo de Agua, Concordia, Departamento de Olancho.

Pleopeltis angusta Humb. et Bonpl. ex Willd. (R. Hernández y R. Moreno 1080, EAP, TEFH). Ubicación. Comunidad de Ojo de Agua, Concordia, Departamento de Olancho.

Pleopeltis crassinervata (Fée) T. Moore (R. Hernández y R. Moreno 1069, TEFH, EAP). Ubicación. Comunidad de Ojo de Agua, Concordia, Departamento de Olancho. 
Polypodium lindenianum Kunze (R. Hernández y R. Moreno 1113, EAP, TEFH).

Ubicación. Sendero Los Olingos, Comunidad de Monte Galán, Municipio de Guaimaca, Departamento de Francisco Morazán.

Polypodium longepinnulatum E. Fourn. (R. Hernández y R. Moreno 1102, EAP, TEFH).

Ubicación. Sendero Los Olingos, Comunidad de Monte Galán, Municipio de Guaimaca, Departamento de Francisco Morazán.

Polypodium plebeium Schltdl. et Cham. (R. Hernández y R. Moreno 1082, EAP, TEFH).

Ubicación. Comunidad de Batideros, Municipio de Orica, Departamento de Francisco Morazán.

Pteris podophylla Sw. (R. Hernández y R. Moreno 1094, EAP, TEFH).

Ubicación. Sendero Los Olingos, Comunidad de Monte Galán, Municipio de Guaimaca, Departamento de Francisco Morazán.

Pteris quadriaurita Retz. (R. Hernández y R. Moreno 1093, EAP, TEFH).

Ubicación. Sendero Los Olingos, Comunidad de Monte Galán, Municipio de Guaimaca, Departamento de Francisco Morazán.

Thelypteris minor (C. Chr.) A.R. Sm. (R. Hernández y R. Moreno 1070, EAP, TEFH).

Ubicación. Comunidad de Ojo de Agua, Concordia, Departamento de Olancho.

Thelypteris scalaris (Christ) Alston (R. Hernández y R. Moreno 1087, EAP, TEFH).

Ubicación. Comunidad de Batideros, Municipio de Orica, Departamento de Francisco Morazán.

Trichomanes radicans Sw. (R. Hernández y R. Moreno 1104, EAP, TEFH).

Ubicación. Sendero Los Olingos, Comunidad de Monte Galán, Municipio de Guaimaca, Departamento de Francisco Morazán.

Vittaria graminifolia Kaulf. (R. Hernández y R. Moreno 1076, EAP, TEFH).

Ubicación. Comunidad de Ojo de Agua, Concordia, Departamento de Olancho.

\section{Discusión}

El presente estudio revelo un total de cinco nuevos registros para Honduras, 34 nuevos registros para el Parque Nacional La Tigra (PNLT) y 46 nuevos registros para la Reserva Biológica Misoco (RBM).

Considerando que el área de estudio es pequeña con respecto al área total de ambas áreas protegidas (La Tigra 24, 040 ha y Misoco 4, 572.3 ha), se puede afirmar que se encontró una alta diversidad. Tal como lo menciona Moran (2004), en la hipótesis de la "Estabilidad del tiempo" justifica la mayor diversidad de helechos en los trópicos, porque estos han sido más estables climáticamente por millones de años, por lo que los períodos de extinción han sido pocos. A la vez es importante dar a conocer que la mayor diversidad se encontró en la zona núcleo de ambas áreas protegidas (senderos La Cascada y Los Olingos), los cuales aún contienen bosque primario como remanentes, pues las actividades antropogénicas son de bajo impacto.

Puede decirse que la riqueza de especies encontradas en el PNLT y la RBM es debido a los microhabitats (rocas, troncos caídos, rizomas de helechos arborescentes, cuevas, y los pequeños cursos de agua) que existen en el área de estudio. De acuerdo a Pincheira (2011), la distribución de las especies en los diferentes microhabitats está relacionada a la estructura del hábitat, humedad, intensidad de luz y disponibilidad de materia orgánica en suelo y troncos.

Es importante resaltar que Asplenium rutaceum se encontró creciendo únicamente sobre las rocas y suelo, con mucha materia orgánica, a la orilla de la quebrada en el sendero Los Olingos y esto puede deberse a que los helechos dependen del agua para su reproducción y sobrevivencia. También se puede indicar que la deficiencia del agua en algún periodo del año puede provocar estrés o muerte para los helechos (Rojas, A., 2016).

La diversidad de helechos encontrada en las zonas de estudio, puede considerarse como un indicador del buen estado que aún tienen estas áreas protegidas, debido a que muchas especies de helechos tienen ciclos de vida cortos, por tanto, su sobrevivencia está determinada por la conservación de condiciones ambientales en estado óptimo, haciéndose adecuado conveniente monitorear datos como precipitación, humedad y temperatura, los cuales pueden variar a lo largo del año por los fenómenos climáticos regulares o cambio climático que afectan al país.

\section{Conclusión}

La aparición de varios de los nuevos registros para Honduras, refleja la escasez de información de su diversidad en helechos y licopodios, por lo que se recomienda inventarios exhaustivos en las áreas protegidas.

La realización de investigaciones como esta, permite conocer y tener información sobre la diversidad de especies que contienen las áreas protegidas, para realizar las gestiones correspondientes, a fin de establecer los mecanismos para realizar su monitoreo y así evitar que algunas especies puedan desaparecer por efecto de los fenómenos climáticos o por actividades antropogénicas.

A la vez es necesario realizar un ordenamiento territorial real, conservación y uso sostenible de la biodiversidad y de esta forma, evitar el cambio de 
uso de suelo que se está produciendo de forma acelerada en la Reserva Biológica de Misoco y en menor grado en el Parque Nacional La Tigra.

\section{Agradecimientos}

Agradecemos al Licenciado Said Laínez, jefe del Departamento de Vida Silvestre (ICF) y al Licenciado Ivo Alvarado, Director Técnico del Parque Nacional La Tigra, quienes proporcionaron transporte y hospedaje para realizar el trabajo de campo en el Parque Nacional La Tigra, al Dr. Armando Ruíz del Museo de Historia Natural de Costa Rica, por la recepción y almacenamiento de los especímenes. Al Dr. Nelson Rene Chávez Hernández, Alcalde de Guaimaca, por la ayuda financiera para el trabajo de campo realizado en la Reserva Biológica Misoco, además del acompañamiento de Roberto Downing, Marlenia Acosta y Dinia Acosta, técnicos del ICF. También agradecemos la ayuda proporcionada por la Dra. Lilian Ferrufino y el Lic. Olvin Oyuela del Herbario TEFH, quienes apoyaron en el secado, almacenamiento y distribución de las muestras hacia el Herbario EAP. Al MSc. Rafael Corrales de la Facultad de Ciencias Espaciales de la UNAH, por la elaboración del mapa del área de estudio. Finalmente agradezco a todos aquellas personas que de una u otra forma nos extendieron su apoyo, nuestros más sinceros agradecimientos.

\section{Referencias}

Congreso Nacional de Honduras (CNH). (5 de agosto de 1987). Decreto número 87-87. págs. 11-12.

Cruz, G., \& Erazo, M. (1977). Análisis de la vegetación del bosque nebulos "La Tigra". Tegucigalpa.

Hernandez, R. (2006). Diversidad de Helechos en el sendero La Cascada del Parque Nacinal La Tigra. Tegucigalpa: USAID/MIRA.

Hernández, R. (2012). Informe Técnico ICFDVS-010-2012. Tegucigalpa.

Hernández, R., Nelson, C., Mejía, T., \& Borjas, G. (2005). Diversidad de helechos en el sendero La Esperanza del Parque Nacional La Tigra. Ceiba, 1-2(46), 29-41.

Instituto Nacional de Conservación y Desarrollo Forestal, Áreas Protegidas y Vida Silvestre (ICF). (2016). Plan Operativo Reserva Bilógica Misoco. Tegucigalpa.

Jimenez, J. (2009). Los helechos del corredor del bosque nuboso de la Baja Verapaz, Guatemala. Santo Domingo de Heredia: INBIO.

Lorea, F., \& Riba, R. (1990). Guía para la recolección y preparación de ejemplares para herbario de Pteridofitas. A.C.: Consejo Nacional de Flora de México.

Mickel, J., \& Smith, A. (2004). The Pteridophytes of Mexico. Memoirs of the New York Botanical Garden, 88, 1-1029.

Moran, R. (2004). A natural history of ferns. Oregon: Timber Press.

Moran, R., \& Riba, R. (1995). Flora Mesoamericana. Volumen 1. Psilotaceae a Salviniaceae. México: UNAM.

Pincheira, U. (2011). Patrones de diversidad de plantas trepadoras y epifitas vasculares en el bosque lluvioso Valdiviano de Sudamérica: una síntesis enre los años 200 y 2010. Phyton, 9-18.

Rojas, A. (2012). Nuevos registros de licopodios y helechos para Honduras y el Parque Nacional Montañas de Celaque. Biodiversidad Neotropical, 8392.

Rojas, A. (2016). Variación en la diversidad y distribución de licófitos y helechos en la Estación Biológica Río Macho y su relación con variaciones climáticas. Tomado de: http://www.observatorioambiental.una. ac.cr/index.php/indicadores-ambientales/tema/357variacion-en-la-diversidad-y-distribucion-de-licofitosy-helechos-en-la-estacion-biologica-rio-macho-y-surelacion-con-variaciones-climaticas, , 13 de Mayo de 2017.

Tribunal Superior de Cuentas (TSC). (2012). Auditoria de gestión ambiental practicada a el Instituto de Conservación forestal sobre la administración del área protegida Parque Nacional La Tigra (PNLT). Tegucigalpa: TSC.

Villeda, E. (2013). Plan de manejo del Parque Nacional La Tigra. Tegucigalpa: ICF. 